\title{
Methodist
}

Double-Hit Ph-Negative Adult B-ALL with MYC and BCL2 Rearrangements and CDKN2A Genetic Abnormalities Have Poor Clinical Outcomes: Report of Two Cases

Betty Chung, DO, MPH, MA, ${ }^{1}$ Tara Miller, MD, ${ }^{1}$ Suyang Hao, MD, ${ }^{1}$ Arthur Zieske, MD,

Youli Zu, MD, PhD, ${ }^{1}$ April Ewton, MD, ${ }^{1}$ Yang O. Huh, MD, ${ }^{2}$ Sai Ravi Pingali, MD, ${ }^{3}$ Swaminathan lyer, $M D^{3,4}$ Anderson Cancer Center, Department of Hematopathology, Division of Pathology and Laboratory Medicine, Houston, TX Houston Methodist Hospital, Department of Hematology and Medical Oncology, Houston, TX

${ }^{4}$ University of Texas MD Anderson Cancer Center, Department of Lymphoma and Myeloma, Division of Cancer Medicine, Houston, TX

\section{Background and Design:}

While much research has focused on the features and poorer prognosis of double/triple-hit diffuse large B-cell lymphoma patients, little is known about the impact of concomitant MYC and $B C L 2$ gene rearrangements on the outcomes of adult B-acute lymphoblastic leukemia (BALL) patients.

Two rare cases of $\mathrm{MYC}+/ \mathrm{BCL} 2+$ double hit B-ALL were identified: one de novo and one likely dedifferentiated from a follicular lymphoma. Clinical characteristics, lab findings, and outcomes were analyzed along with tissue evaluation and cytogenetic studies. Bone marrows were subjected to next generation sequencing to identify gene mutations which may modify prognosis in these double hit acute leukemia patients.
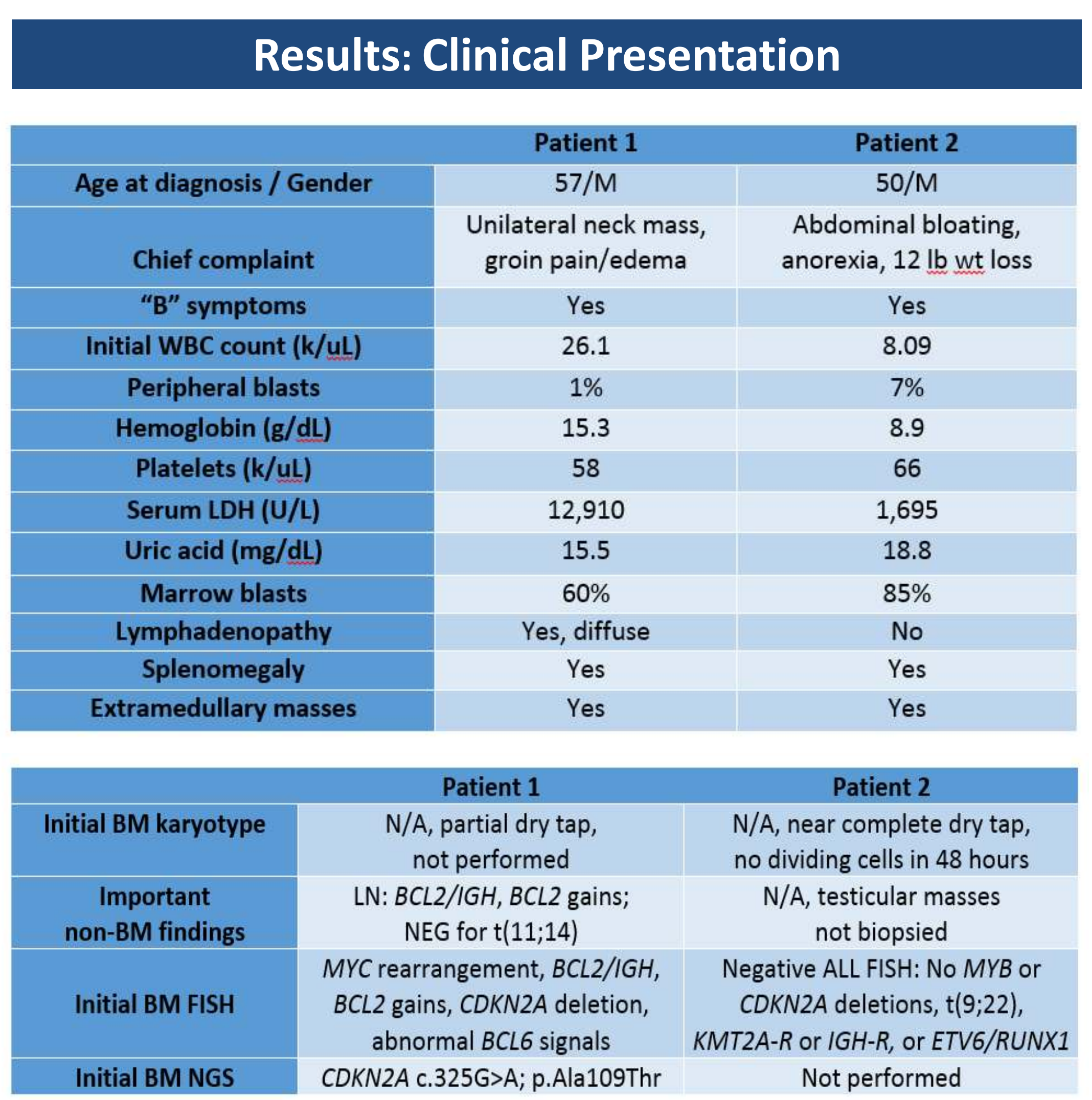

Patient 1: High Grade Follicular Lymphoma \& Double-Hit B-Acute Lymphoblastic Leukemia

- 3 week $\mathrm{h} / \mathrm{o}$ left-sided neck swelling and fatigue, previously treated as infectious

- New onset right scrotal and lower extremity edema and pain

- CT scan: Extensive lymphadenopathy and right hydronephrosis due to mass effect

- Initial concern for undiagnosed CLL/SLL with Richter transformation; Clonal CD5+ B cells

- Inguinal lymph node with grade 3A follicular lymphoma and microfocal B-ALL

- Bone marrow involvement with B-ALL

- No remission; died 9 months after his initial diagnosis



- 2 week h/o abdominal bloating, anorexia, $12 \mathrm{lb}$ weight loss, previously treated as infectious

- Bone marrow involvement with de novo B-ALL and extramedullary masses

- Bilateral testicular masses; h/o undescended right testicle status post-orchiopexy at age 10

- 3 month remission and new onset pararenal mass; transferred for clinical trial

- CD20-positive chest wall mass; died 6 months after his initial diagnosis

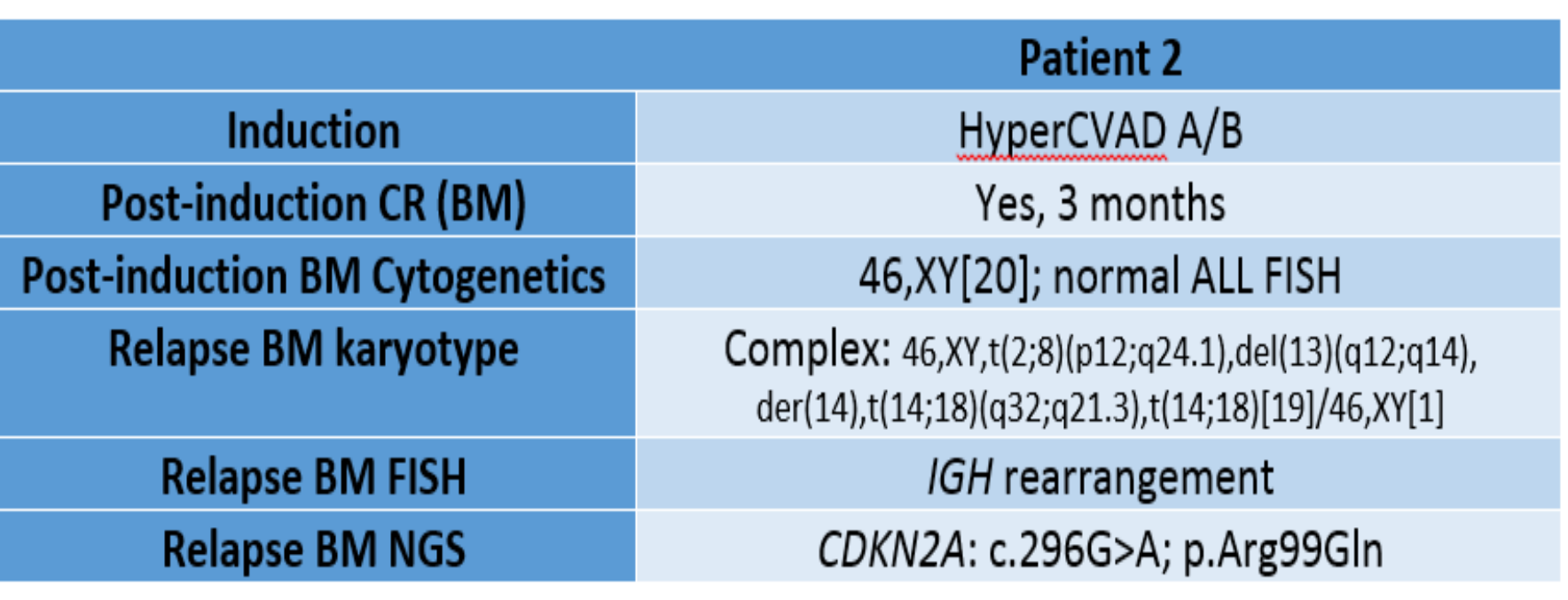

Abstract/Poster\# ALL-207

Discussion

Patient 1:

- $t(14 ; 18)$ subclone likely acquired $t(8 ; 14)$ in the bone marrow where it transformed to B-ALL

- Significance of the clonal CD5-positive B cells in the blood, marrow, and lymph node is unclear

- CDKN2A exon 2 missense mutation prior to induction

- CDKN2A deletion and KIT, ATRX, and STAG2 missense mutations post-induction

Patient 2:

- $\mathrm{t}(2 ; 8)$ and biallelic $\mathrm{t}(14 ; 18)$

- Pararenal mass with B-ALL/B-LBL morphology

- Prior to death, developed a CD20-positive chest wall mass

Both patients had CDKN2A exon 2 missense mutations which may result in $\mathrm{p} 16$ inactivation. This may also result in responsiveness to the novel use of cdk4/cdk6 inhibitors which are currently employed in breast cancer treatment. CDKN2A and MYC deregulation have also been associated with transformation.

\section{Conclusion}

- FISH for MYC, BCL2, and/or BCL6 translocations is not generally performed for leukemia but may help identify poor prognosis double/triple-hit patients

- CDKN2A exon 2 missense mutations and/or CDKN2A deletions may contribute to HyperCVAD refractory disease or rapid relapse in these patients

- It is imperative that we identify additional prognostic and/or predictive biomarkers which may impact disease outcome in adult Ph- B-ALL patients 